\title{
Emerging technologies in solid drug delivery: an interview with Nadia Passerini
}

\begin{abstract}
Nadia Passerini is interviewed by Hannah Makin, Commission Editor. Nadia Passerini is Associate Professor of Pharmaceutical Technology at the University of Bologna (Bologna, Italy). She obtained the degree in Pharmaceutical Chemistry and Technology at the University of Bologna in 1992 and the PhD in Pharmaceutical Science in 1997. Her research focuses on the study of drug delivery systems, developing new technologies and new apparatus for the production of solid dosage forms (microparticles, granules and tablets), which can optimize the bioavailability of drugs according to the specific needs of the therapy. Furthermore, she is interested in the solid-state characterization of the produced delivery systems in order to correlate their physicochemical properties to the in vitro release of the drugs. Currently, her research focuses in particular on the production and characterization of microparticles produced by the spray congealing technology. She is author of over 50 international peer-reviewed publications and over 50 contributions (poster and oral presentations) to national and international conferences.
\end{abstract}

First draft submitted: 12 December 2016; Accepted for publication: 14 December 2016; Published online: 31 January 2017

Keywords: emerging technologies • personalized delivery • small-molecule drugs

\section{First of all, could you tell us a little} about how you first started working in the field of drug delivery?

I started working in the field of drug delivery around 25 years ago, during a 1-year project to obtain my degree in Pharmaceutical Chemistry and Technology from the University of Bologna (Bologna, Italy). The project focused on the investigation of formulation parameters on the release of progesterone from polylactide microspheres prepared using a process based on the solvent evaporation method. This has been my first step into the microparticles world. After so many years, I am still working on microparticulate systems, I have just moved from a solvent evaporation technique to a solvent-free one!

Then, during my $\mathrm{PhD}$, I investigated the application of ultrasounds for the tableting of pharmaceutical powders. In the mid1990 s, ultrasound-assisted compression of materials was well known in the metallurgy, plastic and ceramic industries; however, its use in the pharmaceutical field was quite a new concept. In this respect, my $\mathrm{PhD}$ research project was very innovative and supported the usefulness of new technology for producing tablets able to modify the release of some model drugs.

After my $\mathrm{PhD}$, I wished to gain some experience abroad. A very good friend, Elisabetta Gianasi, was a post doc at the School of Pharmacy in London (the famous 'Square'....), so with her help I got in touch with Professor Duncan Craig. Briefly, I first spent a few months working in his group, and then I won a Marie Curie Fellowship grant, which permitted me to return to Craig's group to continue my research for

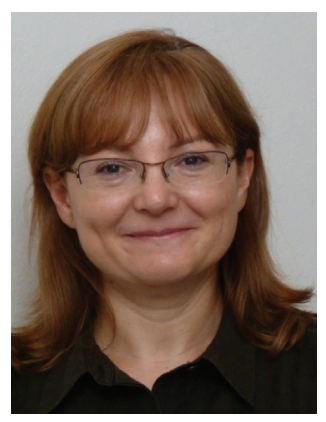

Nadia Passerini Universita degli Studi di Bologna, Department of Pharmacy \& Biotechnology, Via S. Donato 19/2 Bologna 40127, Italy Tel.: +39051 2095613 nadia.passerini@unibo.it 
another year. This experience was very important, as I had the opportunity to receive excellent training in thermoanalysis. Inspired by Craig's mentorship, I have realized the importance of a strong solid-state characterization of dosage forms. It is instrumental to correlate the physicochemical properties of all solid drug delivery systems with their biopharmaceutical ones. At the end of the project, I joined as a Lecturer at the University of Bologna, where I still work, trying to combine my different experiences.

\section{What work are you \& your research group currently focused on \& where do you think this will lead to in the future?}

Since the end of the 1990s, my research is related to the application of different technologies for the development of solid drug delivery systems, which can optimize the bioavailability of drugs according to the therapy's specific needs. We have developed innovative technologies or apparatus for the production of solid dosage forms: in particular, we study the spray congealing technology for the preparation of microparticles (having dimensions in the range of 30-700 microns) and the melt- and steam-granulation for granule manufacturing. In addition, we investigate the effects of formulative and process parameters on the technological and biopharmaceutical properties of solid dosage forms, both manufactured by our innovative techniques and by traditional ones.

Between our current projects, I would like to mention our efforts to develop medicine for pediatric patients. In particular, we are working on designing and manufacturing appropriate solid dosage forms for the administration of praziquantel to children. Praziquantel is an anthelmintic drug widely used in developing countries for the treatment of Schistosomiasis, which is the second most prevalent disease in African children. We have been working on this drug for several years, trying to improve its very low poor water solubility and bioavailability, and to also reduce the high therapeutic doses. We have recently published a paper in International Journal of Pharmaceutics describing the development of flexible and dispersible oral granules containing praziquantel for the treatment of preschool age children [1]. Currently, we are working in collaboration with colleagues at the University of Trieste (Trieste, Italy) on new approaches to enhance the bioavailability of this anthelmintic drug by modifying its solid state.

I hope that our knowledge and efforts lead to the design of an appropriate solid dosage form which, being cheap to produce, is suitable for preschool- and school-aged children living in developing country.
What are the major challenges currently facing researchers in your field of work? Pharmaceutical technology is a field of research which is, by its nature, very close to the pharmaceutical industry. I feel that the major challenge for all university researchers working in drug delivery is to find the right balance between basic academic research, and an industrial-driven one, which needs a quick exploitation of $\mathrm{R} \& \mathrm{D}$ results.

\section{What do you think are the main} advantages of solid dosage forms, such as microparticles, granules \& tablets, over other drug dosage forms?

Generally speaking, it is well known that compared with liquid dosage forms, all the solid dosage forms (tablets, granules, microparticles) have advantages in terms of physical, chemical and microbiological stability of the drug, therefore they have a longer shelflife. Moreover, solid dosage forms are usually cheaper to produce, easy to handle and, if necessary, they can modify the drug release.

In addition, microscale multiparticulate dosage form, such as microparticles and granules, provides further advantages over single-unit systems (tablets): the transport in the GI tract is independent of gastric empting, leading to minor absorption variability. Finally, multiparticulate drug delivery systems allow the preparation of personalized doses for different aged patients; thereby, they are particularly appropriate for children.

\section{Some of your recent work focuses on the} use of solid-lipid microparticles as novel drug delivery systems. What significance does your work in this area hold in the field of drug delivery [2]?

The paper you have cited is the last one we have published on the use of solid-lipid microparticles (SLMs) for oral drug delivery. To me, it represents a nice example of a successful collaboration between research groups working in different disciplines. Some colleagues at the University of Bologna have previously developed an innovative nonradical scavenger that has been used with success in several pathological conditions and disease mechanisms, such as inflammation, neurological disorders, hepatitis and diabetes, all related to an over production of free radicals. However, due to characteristics of the molecule (the breakage of its aliphatic chain by gastric acidity), all the pharmacological treatments were performed by the intraperitoneal route of administration, which is not the patient's favorite one. Thus, our contribution to the project was to design and to produce a 
delivery system that allows the oral administration of the innovative nonradical scavenger while maintaining its therapeutic efficacy. The selected approach involved the investigation of a microparticulate system for the above-mentioned advantages over tablets. In particular, we have developed SLMs due to the favorable biocompatibility and lower toxicity of the lipids compared with many polymers. In addition, most of the excipients used are generally recognized as safe. In general, the most significant contribution of my group to the development of SLMs as drug delivery system is the peculiar technology (i.e., spray congealing), we employ for their production.

\section{What further work do you think needs to be done in order to fully understand the potential of SLMs in drug delivery?}

I totally agree with the opinion recently reported by Scalia et al. in an excellent review on SLMs [3]: SLMs are indeed a very attractive drug delivery system. Some delivery routes, such as the oral one, are more established than others. The investigation of the application of SLMs via other delivery routes may help to fully understand their potential in drug delivery.

\section{Also recently, you investigated whether spray-congealed microparticles could be used to enhance the solubility of certain drugs [4]. Can you tell us a little bit about why you chose to study the uses of spray congealing technologies?}

Briefly, spray congealing technology, also called spray chilling or spray cooling, consists of the atomization of a fluid (solution or suspension of a drug active in a molten carrier) into an environment maintained at a temperature below the carrier melting point. Suitable carriers have relatively low melting points $\left(40-80^{\circ} \mathrm{C}\right)$. The atomization leads to the formation of molten droplets, which then solidify upon cooling, producing the final microparticles. The microparticles are typically ready-to-use, without need of further postprocessing. The microparticles obtained using our apparatus, are usually spherical in shape, have good encapsulation efficiency and possess excellent flowability. Therefore, they are suitable for capsule filling for oral administration. Spray congealing attracted my attention because this technique does not require the use of organic or aqueous solvents and hence is environmentally friendly and less timeand energy-consuming, compared to other methods. In addition, the spray congealing is easy to scale up and potentially suitable for the continuous manufacturing, which is one of the recent key issue for the pharmaceutical industry.
A further advantage of spray congealing is its versatility, due to the fact that both hydrophilic and hydrophobic low melting point carriers can be used. The selection of the suitable carrier permits to modify the dissolution behavior of the active pharmaceutical ingredients: hydrophobic carriers can be employed to control the release of short half-life drugs or to protect a molecule from the gastric environment, while hydrophilic carriers can be utilized to enhance the dissolution rate of poorly water-soluble drugs, as in the paper you have just mentioned [4]. In that work, we have demonstrated that spray congealing technology can be exploited to produce a solid Self-Emulsifying Drug-Delivery System, which is potentially very useful for all drugs belonging to the Class II of the Biopharmaceutical Classification System.

\section{How do you think further research into this area will have an impact in the field of drug delivery?}

In recent decades, a lot of research on the application of spray congealing in the pharmaceutical field has been carried out. I hope that these efforts will lead to an increasing number of commercially available medicines produced using this technology.

Do you have any words of advice you would give to a young scientist envisaging a career in your field of expertise?

My advice is for all young scientists envisaging a career in sciences, not specifically in drug delivery. As I have previously mentioned, I feel that my experience in the UK has been highly rewarding for my career for several reasons. First, I firmly believe that the exposure to different working practices and to a different scientific culture invaluably broadens our perspectives on how research problems can be approached. In addition, I have had the opportunity to build relationships with people from many different countries and to develop collaborations with them, still ongoing after many years. Therefore, my suggestion is to do a post doc in a foreign country, even better in a well-known international group either in Europe (taking advantages of the chances offered by EU programs) or overseas.

\section{Financial \& competing interests disclosure}

The author has no relevant affiliations or financial involvement with any organization or entity with a financial interest in or financial conflict with the subject matter or materials discussed in the manuscript. This includes employment, consultancies, honoraria, stock ownership or options, expert testimony, grants or patents received or pending, or royalties.

No writing assistance was utilized in the production of this manuscript. 


\section{References}

1 Trastullo R, Dolci L, Passerini N, Albertini B. Development of flexible and dispersible oral formulations containing praziquantel for potential schistosomiasis treatment of preschool age children. Int. J. Pharm. 495, 536-550 (2015).

2 Passerini N, Albertini B, Sabatino MD et al. Development of microparticles for oral administration of the nonconventional radical scavenger IAC and testing in an inflammatory rat model. Int. J. Pharm. 512, 126-136 (2016).
3 Scalia S, Young PM, Traini D. Solid lipid microparticles as an approach to drug delivery. Expert Opin. Drug Deliv. 12 (4), 583-599 (2015)

4 Albertini B, Sabatino MD, Melegari C, Passerini N. Formulation of spray congealed microparticles with selfemulsifying ability for enhanced glibenclamide dissolution performance. J. Microencapsul. 32, 181-192 (2015). 